\title{
Dificuldades de Aprendizagem em Meninos e Meninas: Uma Revisão Sistemática com Metanálise
}

Dificultades de Aprendizaje en Niños y Niñas: Una Revisión Sistemática con el Metanálisis Learning Difficulties in Boys and Girls: A Systematic Review with Meta-analysis

Larissa Leal Serafim Rodrigues ORCID: http://orcid.org/0000-0002-8880-0912 Universidade Federal de Campina Grande, Paraíba/Brasil

Nalyson Almeida Rodrigues

ORCID: https://orcid.org/0000-0002-2139-1309 Universidade Federal de Campina Grande, Paraíba/Brasil

Monilly Ramos Araujo Melo

ORCID: https://orcid.org/0000-0001-6496-371X Universidade Federal de Campina Grande, Paraíba/Brasil

\section{Resumo}

Alguns estudos, na área da educação e da psicologia, dedicam-se a tentar compreender e explicar se há diferenças no processo de aprendizagem entre meninos e meninas. Nesse sentido, objetiva-se realizar uma revisão sistemática com metanálise dos estudos que trabalham com a temática das dificuldades de aprendizagem em crianças, buscando investigar e expor o que a literatura atual apresenta sobre as possíveis causas para essa diferença. Para tanto, no mês de janeiro de 2018, foram realizadas buscas nas bases de dados SciELO, LILACS, BVS-PSI, PePSIC e nos periódicos disponíveis na BDTD e CAPES. Os critérios de inclusão utilizados foram: textos que apresentam referência direta ao tema "dificuldades de aprendizagem em crianças"; publicados entre 2013 e 2018; disponíveis em português e inglês; apresentam a realidade brasileira; possuem referencial teórico da psicologia cognitiva e/ou da neuropsicologia; e trabalham o processo de aprendizagem em crianças. Foram analisados 55 estudos e realizado testes de normalidade da amostra e correlação entre variáveis. Os estudos apontam algumas possíveis explicações para o baixo desempenho escolar e a maior dificuldade de aprendizagem dos meninos como: o comportamento externalizante e a baixa autoeficácia, mais observada em meninos; e o contexto social e familiar, que estimulam mais as meninas a serem disciplinadas e responsáveis, qualidades essenciais para o processo de aprendizagem. No que se refere à limitação do estudo, destacam-se as bases de dados, idiomas e período de publicação dos estudos, que não abarcam todos os textos existentes na área.

Palavras-chave: Aprendizagem; Diferenças; Meninos e meninas; Revisão sistemática.

\section{Resumen}

Algunos estudios, en el ámbito de la educación y la psicología, se dedican a intentar comprender y explicar si hay diferencias en el proceso de aprendizaje entre niños y niñas. En este sentido, se pretende realizar una revisión sistemática con metanálisis de los estudios que trabajan con la temática de las dificultades de aprendizaje en niños, buscando investigar y exponer lo que la literatura actual presenta sobre las posibles causas para esa diferencia. Para ello, en el mes de enero de 2018, se realizaron búsquedas en las bases de datos SciELO, LILACS, BVS-PSI, PePSIC y en los periódicos disponibles en BDTD y CAPES. Los criterios de inclusión utilizados fueron: textos que presentan 
referencia directa al tema "dificultades de aprendizaje en niños"; publicados entre 2013 y 2018; disponible en portugués y en inglés; presentan la realidad brasileña; tienen referencial teórico de la psicología cognitiva y/o de la neuropsicología; y trabajan el proceso de aprendizaje en los niños. Se analizaron 55 estudios y realizaron pruebas de normalidad de la muestra y correlación entre variables. Los estudios apuntan algunas posibles explicaciones para el bajo desempeño escolar y la mayor dificultad de aprendizaje de los niños como: el comportamiento externalizante y la baja autoeficacia, más observada en niños; y el contexto social y familiar, que estimulan más a las niñas a ser disciplinadas y responsables, cualidades esenciales para el proceso de aprendizaje. En lo que se refiere a la limitación del estudio, se destacan las bases de datos, idiomas y período de publicación de los estudios, que no abarcan todos los textos existentes en el área.

Palabras-clave: Aprendizaje; Diferencias; Niños y niñas; Revisión sistemática.

\begin{abstract}
Some studies in the area of education and psychology have been dedicated to trying to understand and explain if there are differences in the learning process between boys and girls. In this way, the objective is to perform a systematic review with meta-analysis of the studies that work with the theme of learning difficulties in children, seeking to investigate and present what the current literature presents about the possible causes for this difference. To do so, in January 2018, searches were performed in the databases SciELO, LILACS, BVS-PSI, PePSIC and in the periodicals available in BDTD and CAPES. The inclusion criteria used were: texts that present direct reference to the theme "learning difficulties in children"; published between 2013 and 2018; available in Portuguese and English; present the Brazilian reality; have theoretical references of cognitive psychology and/or neuropsychology; and work the learning process on children. It was analyzed 55 studies and tests of normality of the sample and correlation between variables were performed. The studies point out some possible explanations for the low school performance and the greater difficulty of learning of the boys as: the externalizing behavior and the low self-efficacy, more observed in boys; and the social and family context, which stimulate more girls to be disciplined and responsible, essential qualities for the learning process. Regarding the limitation of the study, the databases, languages and publication period of the studies stand out, which do not cover all the existing texts in the area.
\end{abstract}

Keywords: Learning; Differences; Boys and girls. Systematic review.

\section{Introdução}

A psicologia cognitiva estuda a mente humana de maneira científica, propondo explicações para os aspectos cognitivos envolvidos no processo de aprendizagem. Muitas são as abordagens, dentro da psicologia cognitiva, que estão voltadas a contribuir cientificamente com o estudo da aprendizagem (Costa \& Domingues, 2013). Dentre elas destaca-se a abordagem do processamento de informações, muito utilizada nos estudos voltados à temática da aprendizagem, por estudar como as pessoas utilizam mentalmente aquilo que conhecem e aprendem sobre o mundo, fazendo uso das habilidades mentais específicas que se assentam nas funções executivas (Sternberg, 2010).

O ambiente escolar é um local de socialização em que as crianças aprendem a conviver com outras crianças, respeitar normas, ter autonomia e a solucionar atividades que demandam o uso dos recursos cognitivos e emocionais. Para o desempenho de tais atividades, exigidas com a finalidade de alcançar o objetivo final da aprendizagem, faz-se necessária à determinação dos objetivos, planejamento para executá-los e controle dos passos estabelecidos que são realizados através do desempenho eficiente 
das funções executivas (Corso, Sperb, Jou \& Salles, 2013).

Dias, Menezes e Seabra (2010) definem as funções executivas como sendo um conjunto de processos cognitivos que permite a regulação do comportamento e da própria cognição, possibilitando o engajamento do indivíduo em ações complexas voltadas para algum objetivo, sendo fundamentais ao direcionamento e regulação de habilidades sociais, emocionais e intelectuais. Dentre os processamentos cognitivos que fazem parte das funções executivas, esses autores apontam: o controle de impulsos, a atenção seletiva, incorporação e manipulação das informações relevantes na memória do trabalho, flexibilidade cognitiva e comportamental, monitoramento das atitudes, planejamento e efetivação das ações. Dessa forma, esses processos cognitivos em conjunto permitem que o indivíduo inicie, planeje, sequencie e monitore os seus comportamentos e cognições, sendo fundamentais para $o$ processo de aprendizagem das crianças.

Nesse sentido, para a neuropsicologia cognitiva, a aprendizagem está relacionada à fase de aquisição da memória e atenção, sendo definida como uma mudança de comportamento viabilizada pela plasticidade dos processos neurais cognitivos resultantes de prática ou experiência anterior (Paula, Beber, Baggio \& Petry, 2006). Nesta perspectiva, quando o conjunto de habilidades cognitivas, representada pelas funções executivas, possui alguma alteração, a criança tende a apresentar problemas de planejamento, organização, dificuldades na inibição, na mudança de respostas e na criação de estratégias, assim como pobre memória do trabalho, sendo refletido nas suas dificuldades de aprendizagem (Dias, Menezes \& Seabra, 2010).

A literatura apresenta diversas definições para as dificuldades de aprendizagem, não havendo consenso a respeito de sua elucidação. Dentre elas, Ciasca (2003) apresenta a dificuldade de aprendizagem como uma "Dificuldade Escolar
(DE) que está relacionada especificamente a um problema de ordem e origem pedagógica" (p. 27). Correia e Martins (2005) apresentam a perspectiva orgânica, em que são consideradas "desordens neurológicas que interferem na recepção, integração ou expressão de informação, caracterizando-se, em geral, por uma discrepância acentuada entre o potencial estimado do aluno e a sua realização escolar" (p. 6).

$\mathrm{Na}$ perspectiva neuropsicológica, Salles, Parente e Machado (2004) apresentam as dificuldades de aprendizagem como sendo "um conjunto de desordens sistêmicas e parciais da aprendizagem escolar que surgem como consequência de uma insuficiência funcional de um ou vários sistemas cerebrais" (p. 111). Sendo esses sistemas cerebrais, segundo os mesmos autores, responsáveis por garantir o surgimento de uma ou várias cadeias interligadas dentro da estrutura psicológica no processo de aprendizagem, a ocorrência de falhas nesse processo resulta em uma aprendizagem deficitária.

Dentre os aspectos que tem se destacado ao se avaliar e estudar crianças com dificuldades de aprendizagem é a questão de gênero, em que a maioria das crianças que apresentam tais dificuldades são meninos. Dessa forma, alguns estudos têm se dedicado a tentar compreender e explicar se há diferenças no processo de aprendizagem entre meninos e meninas (Alliprandini, de Lima, de Oliveira \& Schiavoni, 2012; Dal'Igna, 2007; dos Santos \& Soares, 2011; Richartz \& Santana, 2010).

Nesse sentido, dos Santos e Soares (2011) apontam como causas para justificar essa diferença no desempenho escolar, durante o ensino fundamental e médio, o comportamento mais calmo das meninas e o comportamento mais agitado dos meninos, além do tratamento diferenciado do professor direcionado aos alunos em relação ao gênero, visto que comportamentos mais pacientes, atribuídos às meninas, estariam associados a um bom desempenho acadêmico. No entanto, esses autores também levantam a discussão a respeito de alunos que possuem o 
comportamento agitado, considerados "bagunceiros", que não prestam atenção na aula e apresentam melhores desempenhos do que alunas consideradas "quietinhas", demonstrando que é preciso levar em consideração a realidade de cada aluno e o contexto no qual estão inseridos.

Dal'Igna (2007) também traz o comportamento como uma das possíveis causas do baixo desempenho dos meninos quando comparados às meninas. Essa autora aponta que os discursos da sociedade para justificar o baixo desempenho de meninos apresentam o seu comportamento (inseguro, desatento, distraído e desinteressado) como a principal causa, já a justificativa para o baixo desempenho escolar das meninas estaria na sua incapacidade cognitiva.

Nessa perspectiva, Biddulph (2002, citado por Dal'Igna, 2007) explica essa diferença entre meninos e meninas através da biologia, em que essa pode influenciar sobre o desenvolvimento e aprendizagem. Esse autor explica que o efeito da testosterona, hormônio masculino, nos meninos afeta $\mathrm{o}$ seu desenvolvimento e comportamento, o que justificaria o comportamento mais agitado e impulsivo deles. Além disso, afirma que a configuração e $o$ formato do cérebro de meninos e meninas se desenvolvem de maneiras diferentes, afetando seus modos de agir e pensar.

Alguns estudos apontam, ainda, um melhor desempenho dos meninos em relação às meninas no que se refere à disciplina de matemática (Andrade, Franco \& de Carvalho, 2016; de Fátima Simões \& Ferrão, 2005; Menezes-Filho, 2007). Esses estudos justificam tal diferença através dos aspectos culturais da sociedade em que estimulam as meninas, desde cedo, com brincadeiras voltadas ao cuidado e afeto, enquanto os meninos são incentivados em suas brincadeiras a desenvolverem o raciocínio lógico-matemático, estimulando experiências laboratoriais.
Diante do exposto e levando em consideração a escassez de revisões sistemáticas na área da psicologia, esse trabalho tem como objetivo realizar uma revisão sistemática com metanálise dos estudos que trabalham com a temática das dificuldades de aprendizagem em crianças, buscando expor o que a literatura atual apresenta sobre as diferenças de desempenho escolar entre meninos e meninas e as possíveis causas para tais diferenças. Dessa forma, visa contribuir para o conhecimento científico e aplicações sociais na educação.

\section{Método}

Para a realização dessa revisão sistemática com metanálise, foram seguidas as etapas de Cooper (2010) acerca dos sete estágios de planejamento de uma metanálise. O fluxograma na Figura 1 sumariza esses estágios. Ressalta-se que a busca e análise dos estudos foi feita por pares, atendendo a recomendação de que cada artigo seja revisado, independentemente, por mais de um revisor (Medina \& Pailaquén, 2010).

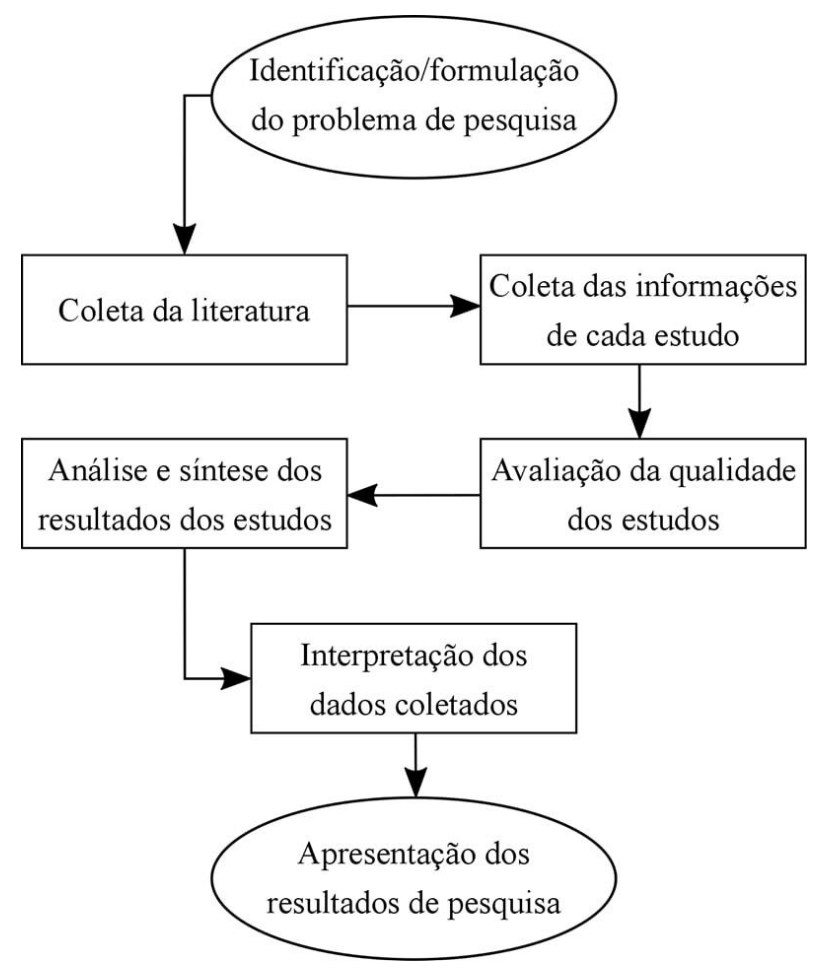

Figura 1. Fluxograma dos estágios de planejamento de uma metanálise 
Dessa forma, no mês de Janeiro do ano de 2018, foram realizadas buscas por textos que abordassem as dificuldades de aprendizagem em crianças, nas bases de dados Scientific Electronic Library Online (SciELO), Literatura Latino-Americana e do Caribe em Ciências da Saúde (LILACS), Biblioteca Virtual em Saúde - Psicologia Brasil (BVSPSI), Periódicos Eletrônicos de Psicologia (PePSIC), nos periódicos disponíveis na Biblioteca Digital Brasileira de Teses e Dissertações (BDTD) e no site da Coordenação de Aperfeiçoamento de Pessoal de Nível Superior (CAPES).

Foram utilizados, com base nas diferenças dos descritores reconhecidos por cada mecanismo de busca textual, os seguintes descritores: Dificuldades de aprendizagem e meninos, Learning Difficulties and boys, Dificuldades de aprendizagem e meninas, Learning Difficulties and girls, Dificuldades de aprendizagem e crianças, Learning Difficulties and children, Desempenho escolar e meninos, School performance and boys, Desempenho escolar e meninas, School performance and girls, Aprendizagem e meninos, Learning and boys, Aprendizagem e meninas, Learning and girls.

Os critérios de inclusão utilizados para a seleção dos estudos foram textos que: 1) apresentam referência direta ao tema "dificuldades de aprendizagem em crianças"; 2) publicados entre os anos de 2013 e 2018; 3) disponíveis em português e inglês; 4) apresentam a realidade brasileira; 5) possuem referencial teórico da psicologia cognitiva e/ou da neuropsicologia; e 6) trabalham o processo de aprendizagem em crianças. Foram desconsiderados estudos em que a amostra apresentou doença crônica, distúrbio psicopatológico ou neuropsicológico e textos que não apresentaram o estudo completo e disponível. Ressalta-se que estudos coincidentes em duas ou mais fontes foram considerados apenas uma vez.

Para realizar a coleta das informações de cada estudo e avaliar a qualidade dos mesmos, foram criadas 22 variáveis: (1)
Título, (2) Análise do Resumo, (3) Idioma do Resumo, (4) Palavra-chave, (5) Justificativa, (6) Objetivo, (7) Tipo de Estudo, (8) Procedimentos Éticos, (9) Amostra, (10) Idade da amostra, (11) Coleta de Dados, (12) Instrumentos, (13) Procedimentos, (14) Análise de dados, (15) Resultados, (16) Limitações, (17) Explicação para as dificuldades de aprendizagem, (18) Relação entre meninos e as dificuldades de aprendizagem, (19) Relação entre meninas e as dificuldades de aprendizagem, (20) Soluções para minimizar as dificuldades de aprendizagem, (21) Soluções por gênero para minimizar as dificuldades de aprendizagem, e (22) Opiniões das crianças sobre as suas próprias dificuldades de aprendizagem. Vale salientar que as variáveis resumo, justificativa e limitações foram criadas baseadas nas normas do PRISMA - Principais Itens para Relatar Revisões sistemáticas e Metanálises (Galvão, Pansani, \& Harrad, 2015).

Para análise e síntese dos resultados dos estudos, foi utilizada a versão 22.0 do software Statistical Package for Social Sciences (SPSS). Para a análise da normalidade das variáveis utilizou-se o teste de Kolmogorov-Smirnov e o teste de Spearman foi utilizado para o cálculo das correlações entre variáveis.

\section{Resultados e discussão}

\section{Análise estatística descritiva}

$\mathrm{Na}$ consulta às bases de dados, foram encontradas 2320 referências potencialmente relevantes que passaram pelo processo de análise através da leitura de seus títulos e resumos, a partir da qual se buscou verificar quais se encaixavam nos critérios de inclusão utilizados, conforme mostra a Figura 2. Dessa forma, após serem examinadas, foram removidas 2265 referências por não estarem em conformidade com os critérios de inclusão utilizados, estando incluso nos removidos textos que não estavam completos e que foram achados mais de uma vez nas diferentes bases de dados, estes últimos sendo contabilizados apenas uma vez. Dentre as referências 
incluídas nessa revisão sistemática estão 24 artigos, 10 teses e 21 dissertações, o que resultou em um número final de 55 estudos, que foram analisados na íntegra.

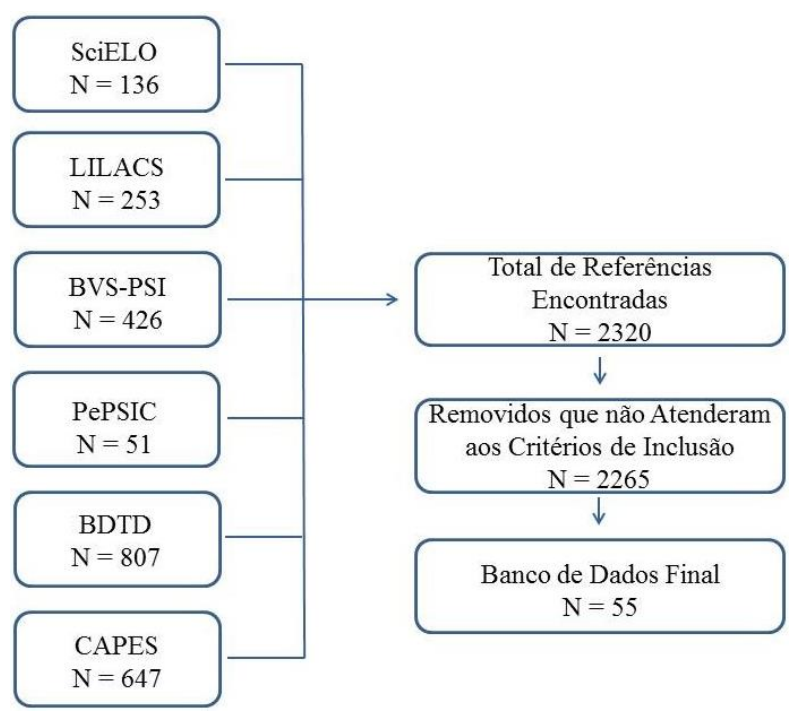

Figura 2. Resultado da busca nas bases de dados

Ao serem efetuadas as análises dos textos incluídos nessa revisão sistemática com metanálise, observou-se que em todos os estudos o título estava de acordo com o achado, descrevendo o que o trabalho se propunha a realizar. Nesse sentido, segundo Imbelloni (2012) o fato de todos os títulos corresponderem aos conteúdos dos respectivos estudos e, principalmente, de haver coerência entre esses títulos e suas respectivas conclusões, indica que os títulos utilizados pelos estudos avaliados apresentaram relevância, concisão e simplicidade.

No que se refere à análise do resumo, $76,4 \%$ dos trabalhos apresentaram os seus resumos completos, em conformidade com as normas do PRISMA (Galvão et al., 2015), apresentando os objetivos, amostra, instrumentos utilizados, análise dos resultados, limitações e conclusões. Ressalta-se que o objetivo do PRISMA é auxiliar os autores na construção de revisões sistemáticas com metanálises, ensaios clínicos randomizados, avaliações de intervenções, dentre outros estudos.

Quanto ao idioma 98,2\% apresentaram o resumo em mais de um idioma, o que possibilita um maior acesso de leitores, sendo os textos divididos da seguinte forma: 45 apresentaram resumos com idiomas em português e inglês, 9 com idiomas português, inglês e espanhol e 1 em português e espanhol. Seguindo essa análise, foi possível constatar que $90,9 \%$ apresentaram as palavras-chave em conformidade com o resumo, o que favorece e facilita a busca na base de dados.

Verificou-se que $94,5 \%$ dos estudos apresentaram a justificativa na introdução, conforme normas do PRISMA (Galvão et al., 2015), sendo descrita e embasada no contexto do que já é conhecido pela literatura e meio científico. Ao analisar os objetivos, notou-se que $94,5 \%$ dos textos apresentavam seus objetivos de forma clara e bem definida. Salienta-se que a elaboração dessas categorias é relevante a fim de verificar se os estudos apresentam seus aspectos estruturais $\mathrm{e}$ metodológicos de maneira completa, com todas as informações necessárias para o leitor.

Com relação ao tipo de estudo $92,7 \%$ dos trabalhos eram estudos empíricos e 7,3\% estudos teóricos, que seriam as revisões de literatura e 1 revisão sistemática. Esses resultados demonstram que os estudos recentes, acerca das dificuldades de aprendizagem em crianças, vêm priorizando uma comprovação prática seja através de experimentos ou observação de determinado contexto. Ressalta-se que, em associação com a teoria, à pesquisa empírica serve para ancorar e comprovar no plano da experiência o que é apresentado teoricamente. Nesse sentido, observa-se que existe uma preocupação, por parte dos pesquisadores, em fornecer dados que fundamentam, refutam e/ou complementam as discussões teóricas sobre as dificuldades de aprendizagem. Além disso, pôde-se notar um baixo número de revisões sistemáticas da literatura, sendo observado em apenas um trabalho, todavia sem metanálise, o que demonstra a necessidade de uma padronização e metodologia mais eficaz na construção de revisões de literatura acerca das dificuldades de aprendizagem em crianças. 
Tratando-se dos aspectos metodológicos dos estudos, observou-se que $74,5 \%$ dos estudos apresentaram os procedimentos éticos utilizados, no entanto, alguns estudos não apresentaram esses procedimentos, mesmo se tratando de pesquisas com seres humanos. Compreende-se por pesquisa com seres humanos aquela que, de forma individual ou coletiva envolva o ser humano, seja direta ou indiretamente. Segundo Ferrari e Rocha (2012) a relevância de apresentar os procedimentos éticos em pesquisas que envolvam seres humanos se deve a necessidade de conduzir ações de pesquisa que possam garantir a segurança, o direito e a dignidade do sujeito envolvido na pesquisa, assim como do pesquisador.

No que se refere à amostra, 98,2\% dos estudos fazem uso de amostra infantil e $89,1 \%$ apresentam a idade da amostra. Ressalta-se que, embora um dos critérios de inclusão seja estudos com crianças, foram encontrados textos nos quais compreendiam as dificuldades de aprendizagem em crianças pela ótica dos professores e/ou pais.

A maioria dos estudos $(98,2 \%)$ apresentaram os procedimentos de coleta de dados de forma clara e bem definida, expondo o período de coleta de dados, local, tempo e instrumentos utilizados. No que se refere a este último $65,5 \%$ fizeram uso em suas pesquisas de instrumentos para coleta de dados. Dessa forma, nota-se que a maioria dos pesquisadores apresentam preocupações quanto à utilização de instrumentos que comprovem suas qualidades psicométricas, como a validade, fidedignidade e normatização (Melo, 2014). Ressalta-se que a utilização de instrumentos com validades psicométricas comprovadas, ou em processo de comprovação, possui uma maior probabilidade de apresentar resultados mais válidos para a construção teórica de um construto.

Com relação à análise de dados 92,7\% dos textos apresentaram os procedimentos de análise de dados utilizados, como o uso de software para análise dos resultados, e $89,1 \%$ apresentaram o tipo de análise utilizada em seus estudos, que seriam análise qualitativa, quantitativa ou ambas no mesmo estudo. Observou-se, ainda, que todos os estudos tiveram os seus resultados alcançados e 54,5\% apresentaram as limitações do estudo na secção de conclusão, de acordo com o PRISMA (Galvão et al., 2015), favorecendo estimar o poder de generalização dos resultados do estudo.

A maioria dos trabalhos analisados (60\%) apresentaram uma explicação geral para as dificuldades de aprendizagem tendo sido, para tal explicação, utilizados os aspectos: comportamentais, emocionais, motivacionais, cognitivos, neurológicos, contexto social, escolar e familiar, conforme mostra Tabela 1. Vale ressaltar que foram encontrados estudos que apresentavam mais de um aspecto, dos citados anteriormente, para explicar as dificuldades de aprendizagem das crianças. Ao analisar se os estudos apresentam alguma explicação sobre a relação entre meninos ou meninas e dificuldades de aprendizagem, verificou-se que $37,5 \%$ tentaram explicar a relação entre meninos e dificuldades de aprendizagem e apenas $16,1 \%$ das pesquisas apresentaram explicações para as possíveis causas das dificuldades de aprendizagem em meninas.

Tabela 1

Distribuição dos artigos por explicações utilizadas

\begin{tabular}{lc}
\hline $\begin{array}{c}\text { EXPLICAÇÕES PARA AS } \\
\text { DIFICULDADES DE } \\
\text { APRENDIZAGEM }\end{array}$ & QUANTIDADE \\
\hline Aspectos Comportamentais & 9 \\
Aspectos Emocionais & 7 \\
Aspectos Motivacionais & 3 \\
Aspectos Cognitivos e & 12 \\
Neurológicos & 15 \\
Contexto Social, Escolar e & \\
Familiar & 46 \\
\hline TOTAL & \\
\hline
\end{tabular}

Os estudos que apresentaram os aspectos comportamentais como possível explicação para o baixo desempenho das crianças e dificuldades de aprendizagem apontam a falta de atenção, desinteresse em 
sala de aula, falta de organização do material escolar e agitação como contribuintes para tais dificuldades (Benetti, Grisard \& Figueiredo, 2014; Borsa, Segabinazi, Stenert, Yates \& Bandeira, 2013; Crepaldi, Correia-Zanini \& Marturano, 2017; Figueiredo, 2013; Fraga, 2016; Guidugli, 2014; Junqueira, 2015; Rezende, 2013; Vivian, Timm \& Souza, 2013).

Dentre esses trabalhos, alguns autores têm destacado a influência dos comportamentos externalizantes e internalizantes no desempenho dos alunos no processo de aprendizagem (Borsa et al., 2013; Guidugli, 2014; Rezende, 2013; Vivian et al., 2013). Nessa perspectiva Guidugli (2014), em seu estudo sobre a relação entre os comportamentos externalizantes e as dificuldades de aprendizagem em crianças, define comportamento externalizante como sendo manifestações comportamentais caracterizadas pela impulsividade, hiperatividade e agressividade, podendo ainda ser manifestado através de um comportamento antissocial e de desafio. Por outro lado, o comportamento internalizante seria definido, por essa mesma autora, como comportamento caracterizado pelo retraimento, ansiedade, depressão, queixas somáticas e afetivas.

Nesse sentido, diante dos resultados desses estudos, foi possível observar que o comportamento externalizante é mais frequentemente relatado e observado em meninos, enquanto que o comportamento internalizante são mais relatados nas meninas (Borsa et al., 2013; Rezende, 2013; Vivian et al., 2013). Diante disso, Vivian, Timm e Souza (2013) e Rezende (2013) destacam que o fato das meninas apresentarem mais comportamentos internalizantes, como problemas afetivos e de ansiedade, faria com que essas não chamassem tanto a atenção de pais e professores para as suas reais dificuldades, o que justificaria o maior encaminhamento de meninos para psicoterapias e reforço escolar.

Alguns estudos afirmam que os aspectos emocionais e motivacionais são essenciais ao processo de aprendizagem das crianças, influenciando no desempenho escolar, assim como sendo considerados fatores resultantes do processo de aprendizagem, em que estariam relacionados às possíveis causas das dificuldades de aprendizagem das crianças (Bezerra, 2014; Conte, Ciasca \& Capeletto, 2016; Figueiredo, 2013; Fraga, 2016; Junqueira, 2015; Osti \& Martinelli, 2014; Silva, Beltrame, Viana, Capistrano \& Oliveira, 2014).

A autoeficácia é referenciada nos estudos sobre motivação como sendo um importante fator para o bom desempenho dos alunos, em que a baixa eficácia seria uma possível causa para explicar as dificuldades de aprendizagem (Junqueira, 2015; Osti \& Martinelli, 2014; Silva et al., 2014). Nesse sentido o estudo de Silva, Beltrame, Viana, Capistrano e Oliveira (2014), que avaliou a autoeficácia dos alunos em função do sexo, demonstrou em seus resultados que as meninas se mostraram mais autoeficazes do que os meninos, o que justificaria o melhor desempenho delas nas atividades escolares.

No que se refere aos aspectos cognitivos e neurológicos, os autores apresentam algumas possíveis causas para as dificuldades de aprendizagem das crianças, tais como: distúrbios e desordens neurológicos e genéticos, assim como déficits nas funções executivas e cognitivas (Andrade, Carvalho, Alves \& Ciasca, 2016; Bezerra, 2014; Carrilho, 2016; Carvalho et al., 2016; Figueiredo, 2013; Fujii, 2015; Lopes, 2016; Machado, 2014; Osti \& Martinelli, 2016; Pazeto, 2016; Pires \& Simão, 2017; Rocha, 2017). Nessa perspectiva Carvalho et al. (2016) apontam como distúrbios neurológicos e genéticos, que podem gerar as dificuldades de aprendizagem, a epilepsia e a encefalopatia não progressiva durante a infância.

Segundo Figueiredo (2013), as desordens neurológicas são responsáveis por interferir na entrada, incorporação e expressão das informações. Tais desordens, para esta autora, envolvem déficits que resultam em problemas de memória, motores, perceptivos, 
metacognitivos, de pensamento e de linguagem, gerando limitações para a aprendizagem de aptidões sociais, leitura, escrita e cálculo.

As funções cognitivas que estariam prejudicadas em crianças que apresentam dificuldades de aprendizagem são apontadas por Andrade, Carvalho, Alves e Ciasca (2016) como sendo: a atenção, memória do trabalho e flexibilidade cognitiva. Pires e Simão (2017) e Carvalho et al. (2016) afirmam que as dificuldades de aprendizagem geradas pelos déficits nas funções executivas e cognitivas podem indicar alguma disfunção do sistema nervoso central da criança, ocasionando falha no processamento de informações refletindo, assim, nas dificuldades apresentadas pelas crianças referentes à leitura, escrita e cálculo. É importante destacar que os estudos, incluídos nessa revisão sistemática com metanálise, que se propuseram a explicar as dificuldades de aprendizagem em crianças levantando como possíveis causas para tais dificuldades os aspectos cognitivos $\mathrm{e}$ neurológicos, não apresentaram nenhuma explicação sobre a diferença de desempenho escolar entre meninos e meninas.

Estudos têm demonstrado que as dificuldades de aprendizagem em crianças são resultado dos contextos: social, representado pela cultura, gênero, raça, situação econômica e interação social; escolar, caracterizado por métodos de ensino ultrapassados e inadequados ao estilo e características dos alunos, escolas sem estruturas adequadas e professores desatualizados e/ou desmotivados; e, por fim, o familiar, que corresponde à interação que se estabelece entre a criança e sua família, os papéis sociais e experiências que ela desenvolve em casa (Bezerra, 2014; Carvalho et al., 2016; Crepaldi et al., 2017; dos Santos \& Araújo Nóbrega, 2016; Figueiredo, 2013; Fraga, 2016; Junqueira, 2015; Lopes, 2016; Macêdo, 2015; Machado, 2014; Osti \& Martinelli, 2014; Pereira et al., 2015; Senkevics \& Carvalho, 2015; Silva, 2017; Tenório, 2013).
Levando em consideração o contexto social, os autores referenciados no parágrafo anterior, apontam como possível explicação para o fato dos meninos apresentarem mais dificuldades de aprendizagem do que as meninas a questão de gênero. Nesse sentido, o papel desempenhado pelas meninas em casa, ajudando nos afazeres domésticos, possuindo deveres e responsabilidades, possibilita que estas amadureçam mais cedo e que desenvolvam atributos como disciplina, organização e responsabilidade, qualidades que a instituição escolar exige dos alunos, resultando em um bom desempenho escolar. Já os meninos, criados sem muitos deveres e responsabilidades, aproveitariam mais as atividades de lazer e não desenvolveriam os atributos de disciplina e responsabilidades, tendo suas vontades individuais acima dos deveres, refletindo no desempenho escolar e nas dificuldades de aprendizagem.

Dos estudos analisados, foi possível observar que apenas 25,5\% apresentaram soluções que visam minimizar as dificuldades de aprendizagem em crianças, independente do gênero. Nessas soluções os autores indicam que sejam necessárias atividades que: estimulem a autoeficácia dos alunos; utilizem jogos e brincadeiras que facilitem o desenvolvimento motor e cognitivo; e incentivem as crianças a compreender $\mathrm{o}$ próprio desempenho e evolução, levando em consideração a visão do aluno sobre o seu processo de aprendizagem (Benetti et al., 2014; Cenci \& Costas, 2013; dos Santos \& Araújo Nóbrega, 2016; Fraga, 2016; Junqueira, 2015; Lopes, 2016; Macêdo, 2015; Machado, 2014; Rocha, 2017; Senkevics \& Carvalho, 2015; Serafin, 2016; Silva et al., 2014; Silva \& Craveiro, 2014).

Além dessas soluções Junqueira (2015), em seu estudo sobre as dificuldades escolares levando em consideração a percepção dos familiares e educadores, ainda aponta a importância de se rever a socialização familiar, sendo necessário que as famílias estimulem as crianças para um melhor desempenho na escola e que esta leve em consideração a história, identidade, 
especificidades e características da criança no planejamento de atividades pedagógicas.

$\begin{array}{ccc}\text { Com } & \text { relação aos estudos que } \\ \text { apresentaram } & \text { soluções levando } & \text { em }\end{array}$
consideração o gênero da criança $(9,1 \%)$, destaca-se a solução apontada por Silva et al., (2014) sugerindo ações que visam o aumento da autoeficácia direcionadas especialmente aos meninos que possuem baixo desempenho escolar. Além disso, Senkevics e Carvalho (2015) sugerem que as famílias repensem a forma como estão criando e educando os meninos, recomendando que estimulem nos meninos qualidades como a disciplina $\mathrm{e}$ responsabilidade, que são essenciais no processo de aprendizagem.

Verificou-se que a grande maioria dos textos analisados $(78,2 \%)$ não consideraram as opiniões das crianças sobre as suas próprias dificuldades de aprendizagem. Conforme aponta Carvalho (2014), a maioria dos estudos que estão sendo produzidos sobre as dificuldades de aprendizagem não estão levando em consideração as perspectivas das próprias crianças sobre suas dificuldades, o que torna esses estudos limitados já que não consideram a concepção dos protagonistas dessas pesquisas, não apresentando soluções voltadas às demandas das crianças.

\section{Análise correlacional}

Tabela 2
Após as análises descritivas, foi realizado o teste de normalidade da amostra (Kolmogorov-Smirnov - KS), no qual foi considerado o valor crítico de 0,181 indicado por Siegel e Castellan Jr (1975), para analisar os valores das estatísticas fornecidos pela a tabela de resultados do KS, considerando uma amostra de 55, com o alpha de 0,05. Nesse sentido, foi observado que todos os valores das estatísticas presentes na tabela estavam acima do valor crítico e que todos os valores de significância foram 0,000 o que resultou em rejeitar a hipótese de normalidade dos dados.

Por se tratar de uma amostra nãoparamétrica, foram empreendidas análises estatísticas através da identificação do Coeficiente de Correlação de Spearman (r). A Tabela 2 apresenta o resultado das correlações significativas entre as variáveis, na qual foram empregados os níveis de significância de $\mathrm{p}<$ 0,01 e $\mathrm{p}<0,05$. Para análise da força das correlações, foi utilizada a classificação de força ou magnitude da relação entre variáveis recomendada por Shimakura (2006). Nessa classificação, $r \leq 0,19$ é considerado uma correlação muito fraca, $0,2 \leq \mathrm{r} \leq 0,39$ fraca, $0,40 \leq \mathrm{r} \leq 0,69$ moderada, $0,70 \leq \mathrm{r} \leq 0,90$ forte, $0,90 \leq \mathrm{r} \leq 1,00$ muito forte. Ressalta-se que na presente revisão, foram encontradas apenas correlações significativas consideradas fracas e moderadas.

\section{Correlações significativas}

\begin{tabular}{lcc}
\hline \multicolumn{1}{c}{ VARIÁVEIS } & r & p \\
\hline Procedimentos de análise - Tipo de análise utilizada & $0,576^{* *}$ & 0,000 \\
Objetivos - Amostra Infantil & $0,567 * *$ & 0,000 \\
Dificuldade Meninos - Dificuldades Meninas & $0,565^{* *}$ & 0,000 \\
Tipo de Estudo - Amostra Infantil & $-0,486^{* *}$ & 0,000 \\
Amostra Infantil - Idade da Amostra & $0,389 * *$ & 0,003 \\
Idade da Amostra - Coleta de Dados & $0,389 * *$ & 0,003 \\
Tipo de Estudo - Instrumentos & $-0,387 * *$ & 0,003 \\
Idade da Amostra - Instrumentos & $0,361^{* *}$ & 0,006 \\
Tipo de Estudo - Idade da Amostra & $-0,352^{* *}$ & 0,008 \\
Procedimentos de Análise - Dificuldades Geral & $0,345^{* *}$ & 0,009 \\
Solução Gênero - Opinião Crianças & $0,294^{*}$ & 0,028 \\
Procedimentos Éticos - Instrumentos & $0,283^{*}$ & 0,035 \\
Instrumentos - Solução para Dificuldades & $-0,283^{*}$ & 0,035 \\
Dificuldades Meninos - Solução Gênero & $0,275^{*}$ & 0,040 \\
Resumo PRISMA - Solução Gênero & $0,273^{*}$ & 0,042 \\
\hline
\end{tabular}

Nota. $\mathrm{r}$ (Coeficiente de Correlação de Spearman-Brown); p (Significância da Correlação); ** = Correlações significativas em nível 0,01 ( $<<0,01) ; *$ = Correlações significativas em nível 0,05 (p < 0,05). 
Diante dos resultados apresentados na Tabela 2, e considerando a classificação das correlações proposta por Shimakura (2006), anteriormente mencionada, observou-se que os estudos possuem uma tendência a trabalharem as dificuldades de aprendizagem dos meninos e meninas simultaneamente, assim como a elucidarem possíveis soluções para tais dificuldades. Referente à correlação entre as variáveis dificuldades de aprendizagem dos meninos e solução por gênero, esperava-se que essa correlação não fosse fraca, visto que ao trabalhar com a temática das dificuldades de aprendizagem em meninos se faz necessário que os estudos apresentem soluções para minimizar tais dificuldades.

Os estudos que explicitaram a idade da amostra tendiam a apresentar os procedimentos de coletas de dados, assim como utilizar escalas ou testes psicológicos. Nesse sentido, a fraca correlação entre as variáveis chama a atenção por serem fatores importantes para o delineamento da metodologia dos estudos empíricos.

Ao analisar os estudos que apresentaram soluções para as dificuldades de aprendizagem, observou-se que estes tendiam a considerar as opiniões das crianças acerca de suas dificuldades de aprendizagem e a não apresentarem um resumo segundo as recomendações do PRISMA. O resultado da correlação entre as variáveis soluções por gênero e opinião das crianças chama a atenção por apresentar uma correlação fraca, sendo esperado uma correlação forte visto que são estudos que trabalham as dificuldades de aprendizagem das crianças, propondo soluções para tais dificuldades sem levarem em consideração as impressões das crianças sobre as suas próprias dificuldades e seu processo de aprendizagem, gerando limitações nesses estudos.

Por fim, observou-se que os estudos que faziam uso de instrumentos tendiam a apresentarem os procedimentos éticos realizados e a não apresentarem soluções para as dificuldades de aprendizagem em crianças. A correlação entre uso de instrumentos e procedimentos éticos deveria ter sido forte já que os estudos que fazem uso de instrumentos são estudos empíricos, nos quais se faz necessário à apresentação dos procedimentos éticos, por serem pesquisas com seres humanos, da mesma forma esperava-se que a correlação entre a variável uso de instrumentos e soluções por gênero fosse forte e positiva por serem estudos voltados a trazerem contribuições para o conhecimento científico e possíveis soluções para as dificuldades de aprendizagem das crianças.

\section{Considerações finais}

As dificuldades de aprendizagem em crianças é um assunto que tem movimentado estudiosos para desenvolverem pesquisas que tragam contribuições para a compreensão de suas causas e proporcionem o surgimento de possíveis soluções para o enfrentamento destas dificuldades. Nesse sentido, este trabalho objetivou realizar uma revisão sistemática com metanálise dos estudos que abordam a temática das dificuldades de aprendizagem em meninos e meninas, buscando compilar os resultados de trabalhos que vem sendo desenvolvidos nos últimos cinco anos sobre o tema.

Ao analisar os estudos incluídos nessa revisão sistemática, observou-se que não há um consenso na literatura analisada sobre uma possível explicação para o fato dos meninos apresentarem mais dificuldades de aprendizagem do que as meninas. Embora não exista, na literatura, uma justificativa para esse fato, os estudos apontam algumas possíveis explicações para essa questão. Dentre as explicações para o baixo desempenho escolar e a maior dificuldade de aprendizagem dos meninos estão: o comportamento externalizante, a baixa autoeficácia, o contexto social e familiar.

No que se refere aos aspectos estruturais e metodológicos dos textos analisados, verificou-se a ausência de informações nas seguintes categorias: resumo, objetivos, procedimentos éticos, procedimentos de coleta e análise de dados e 
limitações. A ausência de informações nessas categorias demonstra uma deficiência estrutural e metodológica dos estudos que estão sendo desenvolvidos, resultando em trabalhos incompletos.

Apesar dessa revisão sistemática com metanálise ter apresentado limitações no que se refere às bases de dados consultadas, idiomas incluídos e período de publicação dos estudos, que não abarcam todos os textos existentes na área, espera-se que esse estudo possa contribuir para o conhecimento científico e aplicações no contexto educacional.
Para finalizar, conforme apresentado ao longo desse trabalho, vale ressaltar a importância de se levar em consideração todos os aspectos que envolvem o processo de aprendizagem das crianças, tais como os aspectos: cognitivos, emocionais, motivacionais e comportamentais, além dos contextos social, familiar e escolar ao qual estão inseridas. Dessa forma, ao levar em consideração tais aspectos, contextos e a concepção da criança sobre seu processo de aprendizagem e suas dificuldades, possibilita que pais, professores e profissionais estimulem as crianças nesse processo e busquem auxiliá-las em suas dificuldades.

\section{Referências}

Alliprandini, P. M. Z., de Lima, C. F., de

Oliveira, D. E. B., \& Schiavoni, A. (2012).

Diferenças entre gênero no uso de estratégias de aprendizagem na educação a distância. IX ANPED Sul: Seminário de Pesquisa em Educação da Região Sul, Caxias do Sul, RS, Brasil, 9.

Andrade, M., Franco, C., \& de Carvalho, J. P. (2016). Gênero e Desempenho em Matemática ao final do Ensino Médio: Quais as relações?. Anais, 1-16.

Andrade, M. J., Carvalho, M. C., Alves, R. J. R., \& Ciasca, S. M. (2016). Desempenho de escolares em testes de atenção e funções executivas: estudo comparativo. Revista Psicopedagogia, 33(101), 123-132.

Recuperado de http://pepsic.bvsalud.org/scielo.php?script= sci_arttext\&pid=S0103$\underline{84862016000200002 \& \ln \mathrm{g}=\mathrm{pt} \& \mathrm{nrm}=\mathrm{iso} .}$.

Araujo, N. K. S. (2015). Análise das dificuldades na resolução de problemas matemáticos por alunos do $5^{\circ}$ ano do ensino fundamental. 140 f., il. Dissertação (Mestrado em ciências e matemática) Universidade Federal de Sergipe.

Recuperado de https://ri.ufs.br/handle/riufs/5174.

Benetti, I. C., Grisard, E., \& Figueiredo, O. (2014). Redes de apoio: Estado, família e escola como contextos promotores de aprendizagem e desenvolvimento. Roteiro,
39(1), 241-260. Recuperado de

http://editora.unoesc.edu.br/index.php/rotei ro/article/view/2838/pdf_26.

Bezerra, M. S. (2014). Dificuldade de aprendizagem e subjetividade: para além das representações hegemônicas do aprender. 157 f., il. Dissertação (Mestrado em Educação) - Universidade de Brasília, Brasília. Recuperado de http://repositorio.unb.br/handle/10482/1777 2.

Borsa, J. C., Segabinazi, J. D., Stenert, F., Yates, D. B., \& Bandeira, D. R. (2013). Caracterização da Clientela Infanto-Juvenil de uma Clínica-Escola de Avaliação Psicológica de uma Universidade Brasileira. Psicologia, 44(1), 73-81. Recuperado de http://revistaseletronicas.pucrs.br/ojs/index. php/revistapsico/article/view/10599/8850.

Carvalho, M. C., Lima, R. F., Souza, G. G. B., Pires, T. C., Pierini, R., Rodrigues, S. D., Simão, A. N. P., \& Ciasca, S. M. (2016). Characterization of school-related problems and diagnoses in a Neuro-Learning Disorder Clinic. Estudos de Psicologia (Campinas), 33(1), 161-171. Recuperado de

http://www.scielo.br/scielo.php?script=sci arttext\&pid=S0103$\underline{166 X 2016000100161 \& \operatorname{lng}=\text { en \&nrm=iso. }}$. 
Carrilho, A. P. N. (2016). Relação entre compreensão leitora e habilidades cognitivas e linguísticas em escolares com distúrbio de aprendizagem. 165 f., il. Tese (Doutorado em Fonoaudiologia) Universidade de São Paulo. Recuperado de http://www.teses.usp.br/teses/disponiveis/2 5/25143/tde-08082016-220203/pt-br.php.

Carvalho, M. G. Q. (2014). As dificuldades de aprendizagem nas vozes das crianças. 236 f. Tese (Doutorado em Ciências Humanas) - Pontifícia Universidade Católica de Goiás, Goiânia. Recuperado de http://tede2.pucgoias.edu.br:8080/handle/te de/708.

Cenci, A., \& Costas, F. A. T. (2013).

Mediação e conceitos cotidianos: os aportes de Feuerstein e Vygotsky para investigar as dificuldades de aprendizagem. Psicologia em Revista, 19(2), 250-270. Recuperado de http://pepsic.bvsalud.org/scielo.php?script= sci_arttext\&pid $=$ S1677$11682013000200008 \& \ln \mathrm{g}=\mathrm{pt} \& \mathrm{nrm}=$ iso.

Ciasca S. M. (org). (2003). Distúrbios de Aprendizagem: proposta de avaliação interdisciplinar. São Paulo: Casa do Psicólogo, p. 220.

Conte, G., Ciasca, S. M., \& Capelatto, I. V. (2016). Relação entre autoconceito e autocontrole comparados ao desempenho escolar de crianças do ensino fundamental. Revista Psicopedagogia, 33(102), 225-234. Recuperado de http://pepsic.bvsalud.org/scielo.php?script= sci_arttext\&pid=S0103$\underline{84862016000300002 \& \operatorname{lng}=\text { pt\&nrm=iso. }}$

Cooper, H. (2010). Research synthesis and meta-analysis: A step-by-step approach (3. ed.) Thousand Oaks, CA: Sage.

Correia, L. M.; Martins, A. P. (2005). Dificuldade de aprendizagem: Que são? Como entendê-las? Biblioteca digital. Coleção educação. Editora Porto. 21 p.

Corso, H. V., Sperb, T. M., Jou, G. I. D., \& Salles, J. F. (2013). Metacognição e funções executivas: relações entre os conceitos e implicações para a aprendizagem. Psicologia: teoria e pesquisa, 29(1), 21-29.
Costa, V. C. A., \& Domingues, S. (2013). Contribuições da Psicologia Cognitiva ao Estudo da Aprendizagem. Anais V SIMPAC - Volume 5 - n. 1 - Viçosa-MG, p. 331-336.

Crepaldi, E. T. S., Correia-Zanini, M. R. G., \& Marturano, E. M. (2017). No limiar do ensino fundamental: estresse, competência e ajustamento em alunos do $1^{\circ}$ ano. Temas em Psicologia, 25(2), 503-515. Recuperado de

http://pepsic.bvsalud.org/scielo.php?script= sci_arttext\&pid=S1413389X2017000200006\&lng=pt\&nrm=iso.

Dal'Igna, M. C. (2007). Desempenho Escolar de Meninos e Meninas: Há Diferença?. Educação em Revista, 46, 241-267.

Dias, N. M., Menezes, A., \& Seabra, A. G. (2010). Alterações das funções executivas em crianças e adolescentes. Estudos Interdisciplinares em Psicologia, 1(1), 8095.

de Fátima Simões, M., \& Ferrão, M. E. (2005). Competência percebida e desempenho escolar em Matemática. Estudos em Avaliação Educacional, 16(32), 25-42.

dos Santos, Aline, \& Soares, Adriana. (2011). A questão do gênero na sala de aula. Ensiqlopédia, Vol. 8, n 1, 23-27.

dos Santos, J. M. S., \& de Araújo Nóbrega, D. G. (2016). Letramento e ludicidade: superando dificuldades da leitura na alfabetização. 83 f., il. Dissertação (Mestrado em Educação) - Universidade Estadual da Paraíba.

Ferrari, R. F., \& Rocha, J. (2012). Da ética a ética em pesquisa envolvendo seres humanos. Revista de Ciências Humanas, 11(16), 25-40.

Figueiredo, M. O. (2013). Análise de um programa de autorregulação para alunos com dificuldades de aprendizagem. Tese (doutorado). Universidade Federal de São Carlos. Programa de Pós-Graduação em Educação Especial. São Carlos. Recuperado de https://repositorio.ufscar.br/handle/ufscar/2 $\underline{923}$. 
Fraga, B. P. (2016). Estudo de caso acerca da influência da atividade física adaptada à disciplina de ciências nas dificuldades de aprendizagem em crianças de uma escola municipal de Porto Alegre. 63 f., il. Dissertação (Mestrado em bioquímica) Universidade Federal do Rio Grande do Sul. Recuperado de http://www.lume.ufrgs.br/handle/10183/14 $\underline{3006}$.

Fujii, R. C. (2015). A relação entre memória de trabalho e competência leitora em crianças em risco de transtorno de aprendizagem do Projeto Acerta: um estudo exploratório. 100 f., il. Dissertação (Mestrado em educação) - Universidade Federal do Rio Grande do Norte.

Recuperado de https://repositorio.ufrn.br/jspui/handle/1234 $\underline{56789 / 20906 .}$.

Galvão, T. F., Pansani, T. S. A., \& Harrad, D. (2015). Principais itens para relatar Revisões sistemáticas e Meta-análises: A recomendação PRISMA. Epidemiologia e Serviços de Saúde, 24(2), 335-342.

Guidugli, P. M. (2014). Efeitos do ensino sistemático e informatizado sobre comportamentos externalizantes concorrentes à aprendizagem de leitura e escrita. 112 f. Dissertação (mestrado) Universidade Estadual Paulista Julio de Mesquita Filho, Faculdade de Ciências. Recuperado de https://repositorio.unesp.br/handle/11449/1 15553? locale-attribute $=$ es.

Imbelloni, L. E. (2012). Títulos de trabalhos científicos: obrigado pela informação contida em seu título. Rev. Bras Anestesiol, 62(2), 140-140.

Junqueira, P. S. P. (2015). Dificuldades escolares: percepções das famílias e dos educadores. Dissertação (mestrado) Universidade Estadual de Campinas, Faculdade de Ciências Médicas, Campinas. Recuperado de http://repositorio.unicamp.br/jspui/handle/R EPOSIP/313686.

Lopes, C. R. M. (2016). A contação de histórias como estratégia pedagógica na estimulação da linguagem oral e escrita de crianças com dificuldades de aprendizagem. 153 f., il. Dissertação (Mestrado em fonoaudiologia) Universidade de São Paulo. Recuperado de http://www.teses.usp.br/teses/disponiveis/2 5/25143/tde-30052017-205503/pt-br.php.

Macêdo, R. G. (2015). Atividades complementares para o desenvolvimento da escrita alfabética com alunos do $2^{\circ}$ ano em estágio pré-silábico. 100 f., il. Dissertação (Mestrado em linguística) - Universidade Federal da Paraíba. Recuperado de http://tede.biblioteca.ufpb.br:8080/handle/t ede/7629.

Machado, A. C. (2014). Avaliação de um programa de resposta à intervenção multinível para estudantes com dificuldades de aprendizagem. 234 f., il. Tese (Doutorado em educação) Universidade Federal de São Carlos. Recuperado de https://repositorio.ufscar.br/handle/ufscar/2 929.

Medina, E. U., \& Pailaquilén, R. M. B. (2010). A revisão sistemática e a sua relação com a prática baseada na evidência em saúde. Revista Latino-Americana de Enfermagem, 18(4), 824-831.

Melo, M. R. A. (2014). Avaliação Psicológica: Evolução e Atualidade. 1. Ed. Curitiba: CRV Editora, v.1000. p. 150.

Menezes-Filho, N. A. (2007). Os determinantes do desempenho escolar do Brasil (pp. 1-31). IFB.

Osti, A., \& Martinelli, S. C. (2014).

Desempenho escolar: análise comparativa em função do sexo e percepção dos estudantes. Educação e Pesquisa, 40(1), 49-59. Recuperado de http://www.scielo.br/scielo.php?script=sci arttext\&pid=S151797022014000100004\&lng=pt\&nrm=iso.

Osti, A., \& Martinelli, S. C. (2016). Perfil de estudantes encaminhados para apoio pedagógico. Psicologia: teoria e prática, 18(3), 168-181. Recuperado de http://pepsic.bvsalud.org/scielo.php?script= sci_arttext\&pid=S151636872016000300013\&lng=pt\&nrm=iso. 
Paula, G. R., Beber, B. C., Baggio, S. B., \& Petry, T. (2006). Neuropsicologia da aprendizagem. Revista Psicopedagogia, 23(72), 224-231.

Pazeto, T. C. B. (2016). Predição de leitura, escrita e matemática no ensino fundamental por funções executivas, na linguagem oral e habilidades iniciais de linguagem escrita na educação infantil. 133 f., il. Tese (Doutorado em distúrbios do desenvolvimento) - Universidade Presbiteriana Mackenzie. Recuperado de http://bdtd.ibict.br/vufind/Record/UPM_8d cec35695e9148209f9cafea7f991c0.

Pereira, S., Santos, J. N., Nunes, M. A., Oliveira, M. G., Santos, T. S., \& MartinsReis, V. O. (2015). Saúde e educação: uma parceria necessária para o sucesso escolar. CoDAS, 27(1), 58-64. Recuperado de http://www.scielo.br/scielo.php?script=sci arttext\&pid=S2317$17822015000100058 \& \operatorname{lng}=\mathrm{pt}$.

Pires, A. B. C., \& Simão, A. N. P. (2017). Avaliação de crianças com indicação de dificuldades de aprendizagem pelo instrumento NEUPSILIN-Inf. Revista Psicopedagogia, 34(104), 148-157. Recuperado de http://pepsic.bvsalud.org/scielo.php?script= sci_arttext\&pid=S0103$\underline{84862017000200005 \& \operatorname{lng}=p t \& n r m=i s o}$.

Rezende, A. R. T. (2013). Dificuldades aritméticas em indivíduos com transtorno do déficit de atenção/hiperatividade: avaliação clínica e por neuroimagem funcional. 165 f., il. Tese (Doutorado em medicina) - Universidade de São Paulo.

Recuperado de http://www.teses.usp.br/teses/disponiveis/5/ 5138/tde-17012014-113849/pt-br.php.

Richartz, T., \& Santana, Z. (2010). Relações de Gênero e Facilidade/Dificuldade de Aprendizagem: A Influência dos Estímulos Recebidos na Educação não Formal e a Repercussão no Desempenho Escolar. Diásporas, Diversidades, Deslocamentos, 1-8.

Rocha, L. B. (2017). Efeitos do Programa de Alfabetização e Raciocínio (PAR) no desempenho acadêmico e na expressão das funções executivas em crianças com dificuldades de aprendizagem. $134 \mathrm{f}$,, il. Dissertação (Mestrado em distúrbios do desenvolvimento) - Universidade Presbiteriana Mackenzie. Recuperado de http://tede.mackenzie.br/jspui/handle/tede/3 179.

Salles, J. F., Parente, M. A. M. P., \& Machado, S. S. (2004). As dislexias de desenvolvimento: aspectos neuropsicológicos e cognitivos. Interações, 9(17), 109-132.

Senkevics, A. S., \& Carvalho, M. P. (2015). Casa, rua, escola: gênero e escolarização em setores populares urbanos. Cadernos de Pesquisa, 45(158), 944-968. Recuperado de http://www.scielo.br/scielo.php?script=sci arttext\&pid=S010015742015000400944\&lng=en\&nrm=iso.

Serafin, E. C. B. (2016). A produção do conhecimento sobre dificuldades de aprendizagem no período de 2001-2011. 215 f., il. Dissertação (Mestrado em educação) - Universidade do Extremo Sul Catarinense. Recuperado de http://repositorio.unesc.net/handle/1/3506.

Shimakura, S. E. (2006). Correlação. CE003 Estatística II. Paraná: Departamento de Estatística da Universidade Federal do Paraná, p. 71-78.

Siegel, S., \& Castellan Jr, N. J. (1975). Estatística não-paramétrica para ciências do comportamento. Artmed Editora.

Silva, B., \& Craveiro, C. (2014). O portfólio como estratégia de avaliação das aprendizagens na educação de infância: considerações sobre a sua prática. Zero- $a$ Seis/Zero a seis, 33-53.

Silva, J., Beltrame, T. S., Viana, M. S., Capistrano, R., \& Oliveira, A. V. P. (2014). Autoeficácia e desempenho escolar de alunos do ensino fundamental. Psicologia Escolar e Educacional, 18(3), 411-420. Recuperado de http://www.scielo.br/scielo.php?script=sci arttext\&pid=S1413$85572014000300411 \& \operatorname{lng}=\mathrm{pt} \& \mathrm{nrm}=$ iso.

Silva, J. C. (2017). A invenção da dificuldade de aprendizagem pela escola. $139 \mathrm{f}$. Dissertação (Programa de Pós-Graduação 
STRICTO SENSU em Educação) -

Pontifícia Universidade Católica de Goiás, Goiânia-GO. Recuperado de

http://tede2.pucgoias.edu.br:8080/handle/te de/3847.

Sternberg, R. J. (2010). Psicologia Cognitiva. Porto Alegre: Artes Médicas. 50 Edição.

Tenório, J. P. (2013). Instructional design and evaluation of reading and writing through educational games for children with learning difficulties (Dissertação
Mestrado). Universidade Federal de São Carlos, São Carlos, SP, Brasil.

Vivian, A. G., Timm, J. S., \& Souza, F. P. (2013). Serviço-escola de psicologia: caracterização da clientela infanto juvenil atendida de 2008 a 2012, em uma Universidade privada do RS. Aletheia, (42), 136-152. Recuperado de http://pepsic.bvsalud.org/scielo.php?script= sci_arttext\&pid=S1413$\underline{03942013000300012 \& \operatorname{lng}=\mathrm{pt} \& \mathrm{nrm}=\mathrm{iso} .}$

\section{Dados sobre os autores:}

- Larissa Leal Serafim Rodrigues: Graduada em Psicologia pela Universidade Federal de Campina Grande - UFCG, onde realizou trabalhos de pesquisa e extensão nas áreas de educação inclusiva e neuropsicologia cognitiva, atuou como pesquisadora e extensionista do Observatório Nacional de Educação Especial - ONEESP (UFSCAR/CNPq) e Inclusão Escolar, Sociabilidade e Sustentabilidade (UFCG/CNPq). Atualmente é pesquisadora do Laboratório de Neuropsicologia Cognitiva e Inovação Tecnológica - LabNEUROCIT (UFCG/CNPq) e do Núcleo de Estudos em Saúde Mental, Educação e Psicometria (UFPB/CNPq).

- Nalyson Almeida Rodrigues: Graduando do curso de Psicologia da Universidade Federal de Campina Grande - UFCG. Atualmente é pesquisador e assessor de dados do Laboratório de Neuropsicologia Cognitiva e Inovação Tecnológica - LabNEUROCIT (UFCG/CNPq) e bolsista de Iniciação Tecnológica.

- Monilly Ramos Araujo Melo: Doutora em Psicologia Cognitiva, pela Universidade Federal de Pernambuco - UFPE. Atualmente é professora efetiva do Departamento de Psicologia da Universidade Federal de Campina Grande - UFCG, pesquisadora e coordenadora do Laboratório de Neuropsicologia Cognitiva e Inovação Tecnológica - LabNEUROCIT (UFCG/CNPq), pesquisadora do Grupo de Estudos e Pesquisas em Psicologia Cognitiva e Cultura (Psicologia Cognitiva/UFPE/CNPq) e do Grupo de Pesquisa em Educação e Psicometria (UFPB/CNPq). 